\title{
A note on the equivalence of three major propagator algorithms for computational stability and efficiency*
}

\author{
Yanlu Ma $\mathrm{Ma}^{1,2, \uparrow}$ Rongjiang Wang ${ }^{3}$ and Huilan Zhou ${ }^{4}$ \\ ${ }^{1}$ Institute for Geology, Mineralogy and Geophysics, Ruhr-University, Bochum 44801, Germany \\ ${ }^{2}$ China Earthquake Networks Center, Beijing 100045, China \\ ${ }^{3}$ GeoForschungsZentrum Potsdam (GFZ), Telegrafenberg, D-14473 Potsdam, Germany \\ ${ }^{4}$ College of Earth Sciences, Graduate University of Chinese Academy of Sciences, Beijing 100049, China
}

\begin{abstract}
It is shown in this note that the three methods, the orthonormalization method, the minor matrix method and the recursive reflection-transmission matrix method are closely related and solve the numerical instability in the original Thomson-Haskell propagator matrix method equally well. Another stable and efficient method based on the orthonormalization and the Langer block-diagonal decomposition is presented to calculate the response of a horizontal stratified model to a plane, spectral wave. It is a numerically robust Thomson-Haskell matrix method for high frequencies, large layer thicknesses and horizontal slownesses. The technique is applied to calculate reflection-transmission coefficients, body wave receiver functions and Rayleigh wave dispersion.
\end{abstract}

Key words: elastic-wave theory; layered media; numerical technique; Rayleigh wave; seismic-wave propagation

CLC number: P315.01 Document code: A

\section{Introduction}

Laterally homogeneous multi-layer models represent an approximation of main characteristics of the three-dimensional heterogeneous Earth structure. A one-dimensional wave speed profile is often a starting point for some more complicated seismic processing methods. In theory, the Thomson-Haskell propagator matrix solution can express the elastodynamic response of a stack of layers due to incident plane waves elegantly, but in practice it may suffer computational instabilities at high frequencies or large layer thickness if waves become evanescent in some layers for a given incident angle. The difficulty was recognized and remedied by replacing the original propagators with its second-order minor matrices by Dunkin (1965) and Thrower (1965). A simple and efficient method which

\footnotetext{
* Received 30 September 2011; accepted in revised form 12 December 2011; published 10 February 2012.

* Corresponding author. e-mail: yanlu.ma@rub.de

(C) The Seismological Society of China, Institute of Geophysics, China Earthquake Administration, and Springer-Verlag Berlin Heidelberg 2012
}

combines the second-order minor method and the Langer block-diagonal decomposition is presented by Chapman (2003). Kennett $(1974,1983,2001)$ derived an recursive algorithm for propagating the generalized reflection-transmission coefficients directly to avoid the difficulties of numerical instabilities. An orthogonalization approach which makes the inner product between two 4D velocity-stress vectors vanished by Gram-Schmidt method was developed by Pitteway (1965) for the radio wave problem and used later in seismology by Chapman and Phinney (1972). The orthogonalization improves the numerical stability of the propagator algorithm, but it requires certain restriction of the propagation step. Wang (1999) proposed another orthogonalization and normalization procedures in Thomson-Haskell propagation loop to overcome the difficulties in these calculations. This kind of orthogonalization means reconstruction of the two velocitystress solutions at each interface so that they express the response respectively to the incident $\mathrm{P}$ and $\mathrm{SV}$ waves within the following layer to be propagated. Thus, the numerical operation between two exponentially increas- 
ing phase terms are avoided and the independence of the two wave solutions can be maintained. The normalization is used to prevent overflow. The orthonormalization method can be easily incorporated with any multidimensional propagator scheme. For example, it has been used for calculating dynamic or static Green's functions of layered elastic (Wang, 1999; Wang et al., 2003), viscoelastic-gravitational (Wang et al., 2006) and poroelastic media (Wang and Kümpel, 2003). In fact, as shown in this note, the two solutions for the computational problems, the minor method and the Kennett's algorithm, are closely related to the orthonormalization method. All of the three can solve the numerical instabilities equally well.
In this note, another efficient and robust method in the Thomson-Haskell propagator matrix formalism, based on the orthonormalization method and the Langer block-diagonal decomposition technique, is presented and used to calculate the reflection-transmission coefficients, synthesis of $\mathrm{P}$ and SV wave receiver functions, and Rayleigh wave dispersion.

\section{Basic equations and matrices}

For the plane P-SV waves in isotropic stratified media, the wave equation and stress-strain relationship can be combined and reduced to first-order differential equations (e.g. Kennett, 2001; Aki and Richards, 2002):

$$
\frac{\mathrm{d}}{\mathrm{d} z}\left(\begin{array}{c}
v_{x} \\
v_{z} \\
\tau_{x z} \\
\tau_{z z}
\end{array}\right)=\mathrm{i} \omega \boldsymbol{A}\left(\begin{array}{c}
v_{x} \\
v_{z} \\
\tau_{x z} \\
\tau_{z z}
\end{array}\right)=\mathrm{i} \omega\left(\begin{array}{cccc}
0 & -p & -\frac{1}{\mu} & 0 \\
-\frac{\lambda p}{\lambda+2 \mu} & 0 & 0 & -\frac{1}{\lambda+2 \mu} \\
\frac{4 \mu(\lambda+\mu) p^{2}}{\lambda+2 \mu}-\rho & 0 & 0 & -\frac{\lambda p}{\lambda+2 \mu} \\
0 & -\rho & -p & 0
\end{array}\right)\left(\begin{array}{c}
v_{x} \\
v_{z} \\
\tau_{x z} \\
\tau_{z z}
\end{array}\right)
$$

here $z$ represents depth, $\left(v_{x}, v_{z}, \tau_{x z}, \tau_{z z}\right)^{\mathrm{T}}$ is the velocity-stress vector on a surface perpendicular to the $z$ axis, $\omega$ and $\rho$ are the angular frequency and density respectively, $p$ is the horizontal slowness or ray parameter, $\lambda$ and $\mu$ are the Lamé moduli. Once the velocity-stress vector $\boldsymbol{x}$ is given at any interface $z_{a}$, its solution at the next interface $z_{b}$ can be obtained via the ThomsonHaskell propagator $\boldsymbol{H}$ :

$$
\boldsymbol{x}\left(z_{b}\right)=\boldsymbol{H} \boldsymbol{x}\left(z_{a}\right)=\boldsymbol{W} \boldsymbol{E} \boldsymbol{W}^{-1} \boldsymbol{x}\left(z_{a}\right) .
$$

Here, the phase matrix $\boldsymbol{E}$ can be decomposed into upgoing and down-going $2 \times 2$ submatrices, i.e.,

$$
\boldsymbol{E}=\left(\begin{array}{cc}
\boldsymbol{E}^{\mathrm{u}} & 0 \\
0 & \left(\boldsymbol{E}^{\mathrm{u}}\right)^{-1}
\end{array}\right)
$$

with

$$
\boldsymbol{E}^{\mathrm{u}}=\left(\begin{array}{cc}
\mathrm{e}^{\mathrm{i} \omega q_{v} d} & 0 \\
0 & \mathrm{e}^{\mathrm{i} \omega q_{p} d}
\end{array}\right),
$$

where $d$ equals to $z_{b}-z_{a}, \pm q_{v}$ and $\pm q_{p}$ are eigenvalues of matrix $\boldsymbol{A}$ for the homogeneous layer between the two interfaces, with $q_{v}=\sqrt{\frac{1}{\beta^{2}}-p^{2}}$ and $q_{p}=\sqrt{\frac{1}{\alpha^{2}}-p^{2}}$. Here $\alpha$ and $\beta$ are the compressional and shear velocities respectively. The matrix $\boldsymbol{W}$, with eigenvectors of matrix $\boldsymbol{A}$ as columns, can be expressed as

$$
\begin{aligned}
& \boldsymbol{W}=\frac{1}{\sqrt{2 \rho}} . \\
& \left(\begin{array}{cccc}
\sqrt{q_{v}} & \frac{p}{\sqrt{q_{p}}} & \sqrt{q_{v}} & \frac{p}{\sqrt{q_{p}}} \\
-\frac{p}{\sqrt{q_{v}}} & \sqrt{q_{p}} & \frac{p}{\sqrt{q_{v}}} & -\sqrt{q_{p}} \\
\frac{2 \mu p^{2}-\rho}{\sqrt{q_{v}}} & -2 \mu p \sqrt{q_{p}} & -\frac{2 \mu p^{2}-\rho}{\sqrt{q_{v}}} & 2 \mu p \sqrt{q_{p}} \\
2 \mu p \sqrt{q_{v}} & \frac{2 \mu p^{2}-\rho}{\sqrt{q_{p}}} & 2 \mu p \sqrt{q_{v}} & \frac{2 \mu p^{2}-\rho}{\sqrt{q_{p}}}
\end{array}\right) .
\end{aligned}
$$

To simplify the propagator matrix $\boldsymbol{H}$, we design the Langer block-diagonalization matrix (e.g. Woodhouse, 1980; Ivansson, 1993; Buchen and Ben-Hador, 1996; Chapman, 2003)

$$
\boldsymbol{L}=\frac{1}{\sqrt{2 \rho}}\left(\begin{array}{cccc}
1 & 0 & 0 & p \\
0 & -p & 1 & 0 \\
0 & 2 \mu p^{2}-\rho & -2 \mu p & 0 \\
2 \mu p & 0 & 0 & 2 \mu p^{2}-\rho
\end{array}\right)
$$

and decompose the propagator $\boldsymbol{H}$ to product of sparse matrices, i.e.,

$$
\boldsymbol{H}=\boldsymbol{L}\left(\boldsymbol{L}^{-1} \boldsymbol{W}\right) \boldsymbol{E}\left(\boldsymbol{W}^{-1} \boldsymbol{L}\right) \boldsymbol{L}^{-1},
$$




$$
\begin{gathered}
\boldsymbol{L}^{-1}=\sqrt{\frac{2}{\rho}}\left(\begin{array}{cccc}
-2 \mu p^{2}+\rho & 0 & 0 & p \\
0 & -2 \mu p & -1 & 0 \\
0 & -2 \mu p^{2}+\rho & -p & 0 \\
2 \mu p & 0 & 0 & -1
\end{array}\right), \\
\boldsymbol{W}^{-1} \boldsymbol{L}=\frac{1}{2}\left(\begin{array}{cccc}
1 & 1 & 0 & 0 \\
0 & 0 & 1 & 1 \\
1 & -1 & 0 & 0 \\
0 & 0 & -1 & 1
\end{array}\right) \cdot\left(\begin{array}{cccc}
\frac{1}{\sqrt{q_{v}}} & 0 & 0 & 0 \\
0 & \sqrt{q_{v}} & 0 & 0 \\
0 & 0 & \frac{1}{\sqrt{q_{p}}} & 0 \\
0 & & \\
\boldsymbol{L}^{-1} \boldsymbol{W} & =\left(\begin{array}{cccc}
0 & 0 & \sqrt{q_{p}}
\end{array}\right) \\
\sqrt{q_{v}} & 0 & 0 & 0 \\
0 & \frac{1}{\sqrt{q_{v}}} & 0 & 0 \\
0 & 0 & \sqrt{q_{p}} & 0 \\
0 & 0 & 0 & \frac{1}{\sqrt{q_{p}}}
\end{array}\right) .\left(\begin{array}{cccc}
1 & 0 & 1 & 0 \\
1 & 0 & -1 & 0 \\
0 & 1 & 0 & -1 \\
0 & 1 & 0 & 1
\end{array}\right)
\end{gathered}
$$

If the three matrices, $\left(\boldsymbol{L}^{-1} \boldsymbol{W}\right), \boldsymbol{E}$, and $\left(\boldsymbol{W}^{-1} \boldsymbol{L}\right)$, are merged into one matrix $\boldsymbol{X}$, the propagator $\boldsymbol{H}$ can be written as $\boldsymbol{L} \boldsymbol{X} \boldsymbol{L}^{-1}$. The $\boldsymbol{X}$ has a block-diagonal form, $\boldsymbol{X}_{v} \oplus \boldsymbol{X}_{p}$, i.e.,

$$
\boldsymbol{X}=\left(\begin{array}{cc}
\boldsymbol{X}_{v} & 0 \\
0 & \boldsymbol{X}_{p}
\end{array}\right)=\left(\begin{array}{cc}
\boldsymbol{X}_{v} & 0 \\
0 & \boldsymbol{I}
\end{array}\right)\left(\begin{array}{cc}
\boldsymbol{I} & 0 \\
0 & \boldsymbol{X}_{p}
\end{array}\right)
$$

where $\boldsymbol{I}$ is the $2 \times 2$ identity matrix, $\boldsymbol{X}_{v}$ and $\boldsymbol{X}_{p}$ are

$$
\boldsymbol{X}_{\zeta}=2\left(\begin{array}{cc}
\cos \left(\omega q_{\zeta} d\right) & \mathrm{i} q_{\zeta} \sin \left(\omega q_{\zeta} d\right) \\
\mathrm{i} \sin \left(\omega q_{\zeta} d\right) / q_{\zeta} & \cos \left(\omega q_{\zeta} d\right)
\end{array}\right)
$$

where the subscript $\zeta$ represents $v$ or $p$.

\section{Numerical problems and solu- tions}

The well-known difficulties involved in the original Thomson-Haskell scheme have already been described by many authors (e.g., Dunkin, 1965; Thrower, 1965; Kind and Odom, 1983; Chapman and Orcutt, 1985; Buchen and Ben-Hador, 1996; Wang, 1999). Here we summarize the two key problems. The first problem is the risk of overflow in the phase matrix $\boldsymbol{E}$ if $q_{p}$ or $q_{v}$ becomes imaginary. The second is the problem of loss of precision. It can be seen in the resultant vector obtained from multiplying the velocity-stress vector $\boldsymbol{x}$ by the propagator $\boldsymbol{H}$ when $\mathrm{P}$ wave becomes evanescent. The result is

$$
\begin{gathered}
\boldsymbol{H} \boldsymbol{x}=\boldsymbol{L}\left(\boldsymbol{L}^{-1} \boldsymbol{W}\right) \boldsymbol{E} \boldsymbol{W}^{-1} \boldsymbol{x}=\frac{1}{\sqrt{2 \rho}} \cdot \\
\left(\begin{array}{c}
\sqrt{q_{v}}\left(\dot{S} \mathrm{e}^{\mathrm{i} \omega q_{v} d}+\grave{S} \mathrm{e}^{-\mathrm{i} \omega q_{v} d}\right)+\frac{p}{\sqrt{q_{p}}} \grave{P} \mathrm{e}^{-\mathrm{i} \omega q_{p} d} \\
\frac{-p}{\sqrt{q_{v}}}\left(\dot{S} \mathrm{e}^{\mathrm{i} \omega q_{v} d}-\grave{S} \mathrm{e}^{-\mathrm{i} \omega q_{v} d}\right)-\sqrt{q_{p}} \grave{P} \mathrm{e}^{-\mathrm{i} \omega q_{p} d} \\
\frac{Z_{p}}{\sqrt{q_{v}}}\left(\dot{S} \mathrm{e}^{\mathrm{i} \omega q_{v} d}-\grave{S} \mathrm{e}^{-\mathrm{i} \omega q_{v} d}\right)+Z_{v} \sqrt{q_{p}} \grave{P} \mathrm{e}^{-\mathrm{i} \omega q_{p} d} \\
Z_{v} \sqrt{q_{v}}\left(\grave{S} \mathrm{e}^{\mathrm{i} \omega q_{v} d}+\grave{S} \mathrm{e}^{-\mathrm{i} \omega q_{v} d}\right)+\frac{Z_{p}}{\sqrt{q_{p}}} \grave{P} \mathrm{e}^{-\mathrm{i} \omega q_{p} d}
\end{array}\right)
\end{gathered}
$$

where $d$ is assumed to be positive, the amplitudes of upgoing and down-going $\mathrm{P}$ and $\mathrm{SV}$ waves come from the vector $(\dot{S}, \dot{S}, \grave{P}, \grave{P})^{\mathrm{T}}$ which equals to $\boldsymbol{W}^{-1} \boldsymbol{x}$, and the exponential decreasing $\mathrm{e}^{\mathrm{i} \omega q_{p} d}$ has been omitted. Given $q_{p}$ is pure imaginary, whether $q_{v}$ is real or imaginary, the magnitudes of items including the exponential growing phase factor $\mathrm{e}^{-\mathrm{i} \omega q_{p} d}$ could be much larger than those including $\mathrm{e}^{-\mathrm{i} \omega q_{v} d}$ and $\mathrm{e}^{\mathrm{i} \omega q_{v} d}$ at high frequencies or large layer thicknesses. The rounding errors in adding or subtracting between $\mathrm{e}^{-\mathrm{i} \omega q_{p} d}$ and other smaller items may result in loss of precision of the latter, 
though they are not exponential small at all. In some extreme cases, if their difference in magnitude is too great, both $\mathrm{e}^{-\mathrm{i} \omega q_{v} d}$ and $\mathrm{e}^{\mathrm{i} \omega q_{v} d}$ items, which represent up-going and down-going SV waves, will be totally lost and only the down-going $\mathrm{P}$ wave will be left in the numerical results when using the original Thomson-Haskell scheme. Consequently, any velocity-stress vector except for those with $\grave{P}=0$ would have the same fate, that is,

$$
\frac{1}{\sqrt{2 \rho}}\left(\begin{array}{c}
\frac{p}{\sqrt{q_{p}}} \\
-\sqrt{q_{p}} \\
Z_{v} \sqrt{q_{p}} \\
\frac{Z_{p}}{\sqrt{q_{p}}}
\end{array}\right) \grave{P} \mathrm{e}^{-\mathrm{i} \omega q_{p} d} .
$$

\subsection{Orthonormalization method}

In the propagator scheme, two independent velocity-stress vectors, represented by a $4 \times 2$ matrix $\boldsymbol{x}$, are required to be propagated. Due to the reflection and transmission at the interfaces, both vectors have generally nonzero $\grave{P}$ components. To maintain the independence of these two solution vectors, Wang (1999) introduced a $2 \times 2$ orthogonalization matrix

$$
\boldsymbol{Q}=\left(\begin{array}{cc}
\grave{P}^{(2)} & -\grave{S}^{(2)} \\
-\grave{P}^{(1)} & \grave{S}^{(1)}
\end{array}\right)
$$

to transform $\boldsymbol{x}$ from right-hand side before multiplied by $\boldsymbol{E}$, then

$$
\boldsymbol{E}(\boldsymbol{x} \boldsymbol{Q})=\boldsymbol{E}\left[\left(\begin{array}{cc}
\dot{S}^{(1)} & \grave{S}^{(2)} \\
\dot{P}^{(1)} & \grave{P}^{(2)} \\
\grave{S}^{(1)} & \grave{S}^{(2)} \\
\grave{P}^{(1)} & \grave{P}^{(2)}
\end{array}\right) \boldsymbol{Q}\right]=\boldsymbol{E}\left(\begin{array}{cc}
\dot{S}^{(1)} \grave{P}^{(2)}-\grave{S}^{(2)} \grave{P}^{(1)} & -\left(\grave{S}^{(1)} \grave{S}^{(2)}-\grave{S}^{(1)} \dot{S}^{(2)}\right) \\
\grave{P}^{(1)} \grave{P}^{(2)}-\grave{P}^{(1)} \grave{P}^{(2)} & -\left(\grave{S}^{(2)} \grave{P}^{(1)}-\grave{S}^{(1)} \dot{P}^{(2)}\right) \\
\grave{S}^{(1)} \grave{P}^{(2)}-\grave{S}^{(2)} \grave{P}^{(1)} & 0 \\
0 & \grave{S}^{(1)} \grave{P}^{(2)}-\grave{S}^{(2)} \grave{P}^{(1)}
\end{array}\right)
$$

where the two column vectors in $\boldsymbol{x}$ are obtained from multiplying $\boldsymbol{W}^{-1}$ with two velocity-stress vectors. By eliminating the $\grave{P}$ component of the first vector to zero, the dominant exponential increasing item $\mathrm{e}^{-\mathrm{i} \omega q_{p} d}$ in the phase matrix $\boldsymbol{E}$ will affect only the second vector, in other words, the loss-of-precision operations with $\mathrm{e}^{-\mathrm{i} \omega q_{p} d}$ in equation (3) are avoided completely in the first vector, thus both contributions from down-going
$\mathrm{SV}$ and $\mathrm{P}$ waves will be preserved respectively within the two vectors during propagation through the layer. It is thanks to the isolation that the risk of overflow can be avoided easily by scaling the two vectors with $\mathrm{e}^{\mathrm{i} \omega q_{v} d}$ and $\mathrm{e}^{\mathrm{i} \omega q_{p} d}$ separately, that is, multiplying with $\boldsymbol{E}^{\mathrm{u}}$ in equation (4) from right-hand side. After orthogonalizing and scaling, we obtain

$$
\boldsymbol{E}(\boldsymbol{x} \boldsymbol{Q}) \boldsymbol{E}^{\mathrm{u}}=\left(\begin{array}{cc}
\mathrm{e}^{2 \mathrm{i} \omega q_{v} d}\left(\grave{S}^{(1)} \grave{P}^{(2)}-\grave{S}^{(2)} \grave{P}^{(1)}\right) & -\mathrm{e}^{\mathrm{i} \omega\left(q_{v}+q_{p}\right) d}\left(\dot{S}^{(1)} \grave{S}^{(2)}-\grave{S}^{(1)} \dot{S}^{(2)}\right) \\
\mathrm{e}^{\mathrm{i} \omega\left(q_{v}+q_{p}\right) d}\left(\grave{P}^{(1)} \grave{P}^{(2)}-\grave{P}^{(1)} \grave{P}^{(2)}\right) & -\mathrm{e}^{2 \mathrm{i} \omega q_{p} d}\left(\grave{S}^{(2)} \dot{P}^{(1)}-\grave{S}^{(1)} \dot{P}^{(2)}\right) \\
\grave{S}^{(1)} \grave{P}^{(2)}-\grave{S}^{(2)} \grave{P}^{(1)} & 0 \\
0 & \grave{S}^{(1)} \grave{P}^{(2)}-\grave{S}^{(2)} \grave{P}^{(1)}
\end{array}\right)
$$

\subsection{Minor matrix method}

The second-order minor of phase matrix $\boldsymbol{E}$ takes the form

$$
\begin{gathered}
\{\boldsymbol{E}\}=\operatorname{diag}\left(\mathrm{e}^{\mathrm{i} \omega\left(q_{p}+q_{v}\right) d}, 1, \mathrm{e}^{\mathrm{i} \omega\left(q_{v}-q_{p}\right) d},\right. \\
\left.\mathrm{e}^{\mathrm{i} \omega\left(q_{p}-q_{v}\right) d}, 1, \mathrm{e}^{-\mathrm{i} \omega\left(q_{p}+q_{v}\right) d}\right),
\end{gathered}
$$

where $\{\cdots\}$ is operation of forming the second-order minors, $\operatorname{diag}(\cdots)$ is a diagonal matrix with the diago- nal elements in the parenthesis. The second-order minor of $\boldsymbol{x}$ is

$$
\{\boldsymbol{x}\}=\left\{\left(\begin{array}{cc}
\dot{S}^{(1)} & \grave{S}^{(2)} \\
\dot{P}^{(1)} & \grave{P}^{(2)} \\
\grave{S}^{(1)} & \grave{S}^{(2)} \\
\grave{P}^{(1)} & \grave{P}^{(2)}
\end{array}\right)\right\}=
$$




$$
\left(\begin{array}{c}
S^{(1)} \dot{P}^{(2)}-\dot{S}^{(2)} \dot{P}^{(1)} \\
\dot{S}^{(1)} \grave{S}^{(2)}-\grave{S}^{(1)} \dot{S}^{(2)} \\
\dot{S}^{(1)} \grave{P}^{(2)}-\grave{S}^{(2)} \grave{P}^{(1)} \\
\dot{P}^{(1)} \grave{S}^{(2)}-\grave{S}^{(1)} \dot{P}^{(2)} \\
\dot{P}^{(1)} \grave{P}^{(2)}-\grave{P}^{(1)} \dot{P}^{(2)} \\
\grave{S}^{(1)} \grave{P}^{(2)}-\grave{S}^{(2)} \grave{P}^{(1)}
\end{array}\right)
$$

Hence it can be seen that all items in equation (16) occur in equation (19), and the matrix $\{\boldsymbol{E}\}\{\boldsymbol{x}\} \mathrm{e}^{\mathrm{i} \omega\left(q_{p}+q_{v}\right) d}$ consists of all the elements of the matrix $\boldsymbol{E}(\boldsymbol{a} Q) \boldsymbol{E}^{\mathrm{u}}$ in equation (17). The scaling factor $\mathrm{e}^{\mathrm{i} \omega\left(q_{p}+q_{v}\right) d}$ (e.g. Panza, 1985; Ivansson, 1993; Buchen and Ben-Hador, 1996) which prevents overflow in a single layer and equals to $\left\{\boldsymbol{E}^{\mathrm{u}}\right\}$, can be dispatched into different matrices, i.e.

$$
\begin{gathered}
\{\boldsymbol{X}\} \mathrm{e}^{\mathrm{i} \omega\left(q_{p}+q_{v}\right) d}= \\
\left(\left\{\boldsymbol{X}_{v} \oplus \boldsymbol{I}\right\} \mathrm{e}^{\mathrm{i} \omega q_{v} d}\right)\left(\left\{\boldsymbol{I} \oplus \boldsymbol{X}_{p}\right\} \mathrm{e}^{\mathrm{i} \omega q_{p} d}\right),
\end{gathered}
$$

where $\boldsymbol{X}$ is given in equation (11), and $\boldsymbol{I}$ is the $2 \times 2$ identity matrix.

Therefore the two methods, the orthonormalization method and the minor method, both employing cross operations between two column vectors, are closely related and solve the computational difficulties equally well.

\subsection{Reflection-transmission matrix method}

If $\boldsymbol{R}_{a}^{\mathrm{d}}$ is the matrix of the generalized reflection coefficients for the layers below the interface $z_{a}$ for downgoing incident $\mathrm{P}$ and $\mathrm{SV}$ waves, a $4 \times 2$ matrix $\boldsymbol{x}_{a}$ whose components are the amplitudes of up-going and downgoing $\mathrm{P}$ and $\mathrm{SV}$ waves can be constructed as

$$
\boldsymbol{x}_{a}=\left(\begin{array}{c}
\boldsymbol{R}_{a}^{\mathrm{d}} \\
\boldsymbol{I}
\end{array}\right)
$$

where $\boldsymbol{I}$ denotes the $2 \times 2$ identity matrix. The 'propagator' for $\boldsymbol{R}_{a}^{\mathrm{d}}$ can be derived from propagating $\boldsymbol{x}_{a}$ from the interfaces $z_{a}$ to $z_{\mathrm{b}}$ by the orthonormalization method. The target is to calculate $\boldsymbol{W}_{b}^{-1} \boldsymbol{W}_{a} \boldsymbol{E} \boldsymbol{x}_{a}$, where $\boldsymbol{E}$ is the phase matrix in equation (3) for the layer between the two interfaces with $d=z_{b}-z_{a}$, and $\boldsymbol{W}_{a}$ and $\boldsymbol{W}_{b}$ are the matrices of eigenvectors for the layers below and above the interface $z_{b}$ respectively. After inserting the scaling matrix $\boldsymbol{E}^{\mathrm{u}}$, we have

$$
\boldsymbol{E} \boldsymbol{x}_{a} \boldsymbol{E}^{\mathrm{u}}=\left(\begin{array}{c}
\boldsymbol{E}^{\mathrm{u}} \boldsymbol{R}_{a}^{\mathrm{d}} \boldsymbol{E}^{\mathrm{u}} \\
\boldsymbol{I}
\end{array}\right)
$$

As the product $\boldsymbol{W}_{b}^{-1} \boldsymbol{W}_{a}$ takes the form (e.g. Kennett, 1974, 1983, 2001)

$$
\boldsymbol{W}_{b}^{-1} \boldsymbol{W}_{a}=\left(\begin{array}{cc}
\boldsymbol{T}_{b}^{\mathrm{u}}-\boldsymbol{R}_{b}^{\mathrm{d}}\left(\boldsymbol{T}_{b}^{\mathrm{d}}\right)^{-1} \boldsymbol{R}_{b}^{\mathrm{u}} & \boldsymbol{R}_{b}^{\mathrm{d}}\left(\boldsymbol{T}_{b}^{\mathrm{d}}\right)^{-1} \\
-\left(\boldsymbol{T}_{b}^{\mathrm{d}}\right)^{-1} \boldsymbol{R}_{b}^{\mathrm{u}} & \left(\boldsymbol{T}_{b}^{\mathrm{d}}\right)^{-1}
\end{array}\right)
$$

where $\boldsymbol{R}_{b}^{\mathrm{d}}, \boldsymbol{T}_{b}^{\mathrm{d}}, \boldsymbol{R}_{b}^{\mathrm{u}}$ and $\boldsymbol{T}_{b}^{\mathrm{u}}$ are the $2 \times 2$ matrices of interface reflection and transmission coefficients at $z_{b}$, multiplying together the two matrices in equations (22) and (23) yields

$$
\begin{gathered}
\boldsymbol{W}_{b}^{-1} \boldsymbol{W}_{a}\left(\boldsymbol{E} \boldsymbol{x}_{a} \boldsymbol{E}^{\mathrm{u}}\right)= \\
\left(\begin{array}{c}
\boldsymbol{T}_{b}^{\mathrm{u}} \boldsymbol{E}^{\mathrm{u}} \boldsymbol{R}_{a}^{\mathrm{d}} \boldsymbol{E}^{\mathrm{u}}+\boldsymbol{R}_{b}^{\mathrm{d}}\left(\boldsymbol{T}_{b}^{\mathrm{d}}\right)^{-1}\left(\boldsymbol{I}-\boldsymbol{R}_{b}^{\mathrm{u}} \boldsymbol{E}^{\mathrm{u}} \boldsymbol{R}_{a}^{\mathrm{d}} \boldsymbol{E}^{\mathrm{u}}\right) \\
\left(\boldsymbol{T}_{b}^{\mathrm{d}}\right)^{-1}\left(\boldsymbol{I}-\boldsymbol{R}_{b}^{\mathrm{u}} \boldsymbol{E}^{\mathrm{u}} \boldsymbol{R}_{a}^{\mathrm{d}} \boldsymbol{E}^{\mathrm{u}}\right)
\end{array}\right) .
\end{gathered}
$$

If the orthogonalizing matrix $Q$ is selected as

$$
\boldsymbol{Q}=\left(\boldsymbol{I}-\boldsymbol{R}_{b}^{\mathrm{u}} \boldsymbol{E}^{\mathrm{u}} \boldsymbol{R}_{a}^{\mathrm{d}} \boldsymbol{E}^{\mathrm{u}}\right)^{-1} \boldsymbol{T}_{b}^{\mathrm{d}}
$$

the matrix of reflection coefficients $\boldsymbol{R}_{b}^{\mathrm{d}}$ for the layers below $z_{b}$ can be obtained by multiplying the matrix $\boldsymbol{Q}$ from the right-hand side, i.e.,

$$
\boldsymbol{x}_{b}=\boldsymbol{W}_{b}^{-1} \boldsymbol{W}_{a}\left(\boldsymbol{E} \boldsymbol{x}_{a} \boldsymbol{E}^{u}\right) \boldsymbol{Q}=\left(\begin{array}{c}
\boldsymbol{R}_{b}^{\mathrm{d}}+\boldsymbol{T}_{b}^{\mathrm{u}} \boldsymbol{E}^{\mathrm{u}} \boldsymbol{R}_{a}^{\mathrm{d}} \boldsymbol{E}^{\mathrm{u}}\left(\boldsymbol{I}-\boldsymbol{R}_{b}^{\mathrm{u}} \boldsymbol{E}^{\mathrm{u}} \boldsymbol{R}_{a}^{\mathrm{d}} \boldsymbol{E}^{\mathrm{u}}\right)^{-1} \boldsymbol{T}_{b}^{\mathrm{d}} \\
\boldsymbol{I}
\end{array}\right)=\left(\begin{array}{c}
\boldsymbol{R}_{b}^{\mathrm{d}} \\
\boldsymbol{I}
\end{array}\right)
$$

Hence the recursive scheme (e.g. Kennett, 1974, 1983; Müller, 1985; Kennett, 2001)

$$
\boldsymbol{R}_{b}^{\mathrm{d}}=\boldsymbol{R}_{b}^{\mathrm{d}}+\boldsymbol{T}_{b}^{\mathrm{u}} \boldsymbol{E}^{\mathrm{u}} \boldsymbol{R}_{a}^{\mathrm{d}} \boldsymbol{E}^{\mathrm{u}}\left(\boldsymbol{I}-\boldsymbol{R}_{b}^{\mathrm{u}} \boldsymbol{E}^{\mathrm{u}} \boldsymbol{R}_{a}^{\mathrm{d}} \boldsymbol{E}^{\mathrm{u}}\right)^{-1} \boldsymbol{T}_{b}^{\mathrm{d}}
$$

is produced from the process of orthogonalizing and scaling. On the other hand, the orthogonalization matrix $\boldsymbol{Q}$ can be expressed by the matrices of reflection- transmission coefficients as in equation (25), and the final result $\boldsymbol{x}_{b}$ can also be arrived directly by using the recursive scheme in equation (27), that is, not through the procedures of orthogonalization and normalization.

Although the orthonormalization method and the reflection-transmission matrix algorithm are motivated by totally different physical insights, they are essential- 
ly equivalent in mathematics on solving the numerical problems occurred in the original Thomson-Haskell propagator method. However, except the additional procedures for orthogonalizing and normalizing, the formalism of the original propagator method is respected in the orthonormalization method. Another difference of the two methods lies in calculations of the intermediate matrices of reflection-transmission coefficients. In recursive algorithm, all of $\boldsymbol{R}_{a}^{\mathrm{d}}$ at every interface $z_{a}$ should be computed in order to iterate uninterruptedly, but in the orthonormalization method, the procedures for orthogonalizing and scaling become indispensable only for decaying $\mathrm{P}$ or $\mathrm{SV}$ waves. As more floating operations are required by these procedures, it should be omitted for efficiency if possible.

$$
\boldsymbol{W}_{1}\left(\begin{array}{cc}
R_{v v}^{\mathrm{d}} & R_{p v}^{\mathrm{d}} \\
R_{v p}^{\mathrm{d}} & R_{p p}^{\mathrm{d}} \\
1 & 0 \\
0 & 1
\end{array}\right)=\boldsymbol{H}\left(z_{2}, z_{3}\right) \cdots \boldsymbol{H}\left(z_{m-1}, z_{m}\right) \boldsymbol{W}_{m}\left(\begin{array}{cc}
0 & 0 \\
0 & 0 \\
T_{v v}^{\mathrm{d}} & T_{p v}^{\mathrm{d}} \\
T_{v p}^{\mathrm{d}} & T_{p p}^{\mathrm{d}}
\end{array}\right)
$$

where the first subscript in reflection-transmission coefficients indicates the incident wave type and the second for generated wave type. The superscript ' $d$ ' means down-going incident wave. Let $\boldsymbol{R}_{2, m}^{\mathrm{d}}$ and $\boldsymbol{T}_{2, m}^{\mathrm{d}}$ represent the generalized reflection and transmission coefficients between the interfaces $z_{2}$ and $z_{m}$ respectively, i.e.

$$
\begin{aligned}
& \boldsymbol{R}_{2, m}^{\mathrm{d}}=\left(\begin{array}{cc}
R_{v v}^{d} & R_{p v}^{\mathrm{d}} \\
R_{v p}^{\mathrm{d}} & R_{p p}^{\mathrm{d}}
\end{array}\right), \\
& \boldsymbol{T}_{2, m}^{\mathrm{d}}=\left(\begin{array}{ll}
T_{v v}^{\mathrm{d}} & T_{p v}^{\mathrm{d}} \\
T_{v p}^{\mathrm{d}} & T_{p p}^{\mathrm{d}}
\end{array}\right),
\end{aligned}
$$

where the first subscript ' 2 ' and the superscript ' $d$ ' indicate the down-going waves incident at $z_{2}$. A bottom-up approach is adopted here to perform the calculations. The program-ready procedure of the approach is shown below.

1) Start the propagation with

$$
\boldsymbol{x}_{m}=\boldsymbol{W}_{m}\left(\begin{array}{cc}
0 & 0 \\
0 & 0 \\
1 & 0 \\
0 & 1
\end{array}\right), \quad \boldsymbol{T}=\left(\begin{array}{cc}
1 & 0 \\
0 & 1
\end{array}\right)
$$

\section{Applications}

\subsection{Reflection and transmission coefficients of a stack of layers}

Following Chapman (2003), we calculate reflectiontransmission coefficients by the method that combines the Langer decomposition and the orthonormalization. Let us consider a stack of layers bounded by two half spaces. The layers are numbered top-down with the half space at the top as the first layer. The interfaces $z_{2}, z_{3}, \cdots, z_{m}$ with $z_{i}$ at the top of $i$ th layer increase upward. The $m$ th layer is the half space at the bottom. The down-going $\mathrm{P}$ and $\mathrm{SV}$ plane wave incident at $z_{2}$. The generalized reflection and transmission coefficients are related by a sequence of Thomson-Haskell propagators and eigenvector matrices $\boldsymbol{W}_{1}$ and $\boldsymbol{W}_{m}$, i.e.,
2) Loop for $i=m-1$ down to 2

$$
\boldsymbol{x}_{i} \leftarrow \boldsymbol{L}_{i}^{-1} \boldsymbol{x}_{i+1}
$$

(a) if $p<\frac{1}{\alpha_{i}}$ then $\boldsymbol{x}_{i} \leftarrow \boldsymbol{X}_{i} \boldsymbol{x}_{i}$

(b) else $\boldsymbol{x}_{i} \leftarrow\left(\boldsymbol{W}_{i}^{-1} \boldsymbol{L}_{i}\right) \boldsymbol{x}_{i}$; build a $2 \times 2$ matrix $\boldsymbol{Q}=\boldsymbol{x}_{i}(3: 4,1: 2)^{-1} ; \quad \boldsymbol{x}_{i} \leftarrow \boldsymbol{x}_{i} \boldsymbol{Q} ; \quad \boldsymbol{T} \leftarrow \boldsymbol{T} \boldsymbol{Q} ; \quad \boldsymbol{x}_{i}(1: 2$, $1: 2) \leftarrow \boldsymbol{E}_{i}^{\mathrm{u}} \boldsymbol{x}_{i}(1: 2,1: 2) \boldsymbol{E}_{i}^{\mathrm{u}}$ with $d=z_{i}-z_{i+1}$ in $\boldsymbol{E}_{i}^{\mathrm{u}}$; $\boldsymbol{T} \leftarrow \boldsymbol{T} \boldsymbol{E}_{i}^{\mathrm{u}} ; \quad \boldsymbol{x}_{i} \leftarrow\left(\boldsymbol{L}_{i}^{-1} \boldsymbol{W}_{i}\right) \boldsymbol{x}_{i}$

$$
\boldsymbol{x}_{i} \leftarrow \boldsymbol{L}_{i} \boldsymbol{x}_{i}
$$

3) Determine the amplitudes of P and SV waves in the half space at the top of the stack.

$$
\boldsymbol{x}_{1} \leftarrow \boldsymbol{L}_{1}^{-1} \boldsymbol{x}_{2} ; \boldsymbol{x}_{1} \leftarrow\left(\boldsymbol{W}_{1}^{-1} \boldsymbol{L}_{1}\right) \boldsymbol{x}_{1}
$$

4) Calculate the generalized reflection-transmission coefficients from $\boldsymbol{T}$ and the partitions of $\boldsymbol{x}_{1}$.

$$
\begin{gathered}
\boldsymbol{R}_{2, m}^{\mathrm{d}} \leftarrow \boldsymbol{x}_{1}(1: 2,1: 2) \boldsymbol{x}_{1}(3: 4,1: 2)^{-1} ; \\
\boldsymbol{T}_{2, m}^{\mathrm{d}} \leftarrow \boldsymbol{T} \boldsymbol{x}_{1}(3: 4,1: 2)^{-1}
\end{gathered}
$$

where ' $\leftarrow$ ' denotes update to, and $\boldsymbol{x}_{i}\left(r_{1}: r_{2}, c_{1}: c_{2}\right)$ represents a submatrix of $\boldsymbol{x}_{i}$ with rows range from $r_{1}$ to $r_{2}$, and columns range from $c_{1}$ to $c_{2}$. When a specific slowness $p$ and angular frequency $\omega$ produce a channel wave mode for the truncated media between the interfaces $z_{i}$ and $z_{m}$, the sub-matrix $\boldsymbol{x}_{i}(3: 4,1: 2)$ becomes 
singular. Such combination of $p$ and $\omega$ should be avoided in the above approach. A Matlab implementation of this algorithm to calculate the generalized reflectiontransmission coefficients of a stack of layers is accompanied with the paper.

\section{2 $\mathrm{P}$ and $\mathrm{SV}$ waves receiver functions}

Here we consider a similar layer system as in section 4.1, except the first layer, the half space at the top, is replaced by the air. The stress components at the free interface $z_{2}$ must vanish. The plane $\mathrm{P}$ and SV waves incident at interface $z_{m}$ and propagate upward. At first, a top-down approach is adopted, i.e.

$$
\begin{gathered}
\left(\begin{array}{c}
\boldsymbol{V}_{2}^{-1} \\
\boldsymbol{R}_{m, 2}^{\mathrm{u}} \boldsymbol{V}_{2}^{-1}
\end{array}\right)=\boldsymbol{W}_{m}^{-1} \boldsymbol{H}\left(z_{m}, z_{m-1}\right) . \\
\boldsymbol{H}\left(z_{m-1}, z_{m-2}\right) \cdots \boldsymbol{H}\left(z_{3}, z_{2}\right)\left(\begin{array}{cc}
1 & 0 \\
0 & 1 \\
0 & 0 \\
0 & 0
\end{array}\right),
\end{gathered}
$$

where $\boldsymbol{R}_{m, 2}^{\mathrm{u}}$ is the generalized reflection coefficients between the interfaces $z_{m}$ and $z_{2}$ with up-going waves incident at the interface $z_{m}, \boldsymbol{V}_{2}$ is the velocities at the free interface $z_{2}$, i.e.

$$
\begin{gathered}
\boldsymbol{R}_{m, 2}^{\mathrm{u}}=\left(\begin{array}{cc}
\boldsymbol{R}_{v v}^{\mathrm{u}} & \boldsymbol{R}_{p v}^{\mathrm{u}} \\
\boldsymbol{R}_{v p}^{\mathrm{u}} & \boldsymbol{R}_{p p}^{\mathrm{u}}
\end{array}\right), \\
\boldsymbol{V}_{2}=\left(\begin{array}{cc}
V_{v x} & V_{p x} \\
V_{v z} & V_{p z}
\end{array}\right) .
\end{gathered}
$$

The procedure of the top-down approach is similar to the procedure in section 4.1 . The only difference is that the two vectors are made orthogonal with respect to the up-going waves instead of the down-going waves, that is, $\boldsymbol{Q}$ is selected to be $\boldsymbol{x}_{i}(1: 2,1: 2)^{-1}$ now. From the viewpoint of mathematics, the process of orthogonalizing and scaling is the multiplications to both sides of equation (30) from right by the matrices $\boldsymbol{Q}_{k_{i}}$ and $\boldsymbol{E}_{k_{i}}^{\mathrm{u}}$ at the layers $k_{1}, k_{2}, \cdots, k_{n}$. When the $m$ th layer is arrived, the final resultant product can be written as

$$
\left(\begin{array}{c}
\boldsymbol{U} \\
\boldsymbol{D}
\end{array}\right)=\left(\begin{array}{c}
\boldsymbol{V}_{2}^{-1} \boldsymbol{Q}_{k_{1}} \boldsymbol{E}_{k_{1}}^{\mathrm{u}} \boldsymbol{Q}_{k_{2}} \boldsymbol{E}_{k_{2}}^{\mathrm{u}} \cdots \boldsymbol{Q}_{k_{n}} \boldsymbol{E}_{k_{n}}^{\mathrm{u}} \\
\boldsymbol{R}_{m, 2}^{\mathrm{u}} \boldsymbol{V}_{2}^{-1} \boldsymbol{Q}_{k_{1}} \boldsymbol{E}_{k_{1}}^{\mathrm{u}} \boldsymbol{Q}_{k_{2}} \boldsymbol{E}_{k_{2}}^{\mathrm{u}} \cdots \boldsymbol{Q}_{k_{n}} \boldsymbol{E}_{k_{n}}^{\mathrm{u}}
\end{array}\right)
$$

and

$$
\boldsymbol{T}=\boldsymbol{Q}_{k_{1}} \boldsymbol{E}_{k_{1}}^{\mathrm{u}} \boldsymbol{Q}_{k_{2}} \boldsymbol{E}_{k_{2}}^{\mathrm{u}} \cdots \boldsymbol{Q}_{k_{n}} \boldsymbol{E}_{k_{n}}^{\mathrm{u}}
$$

Therefore $\boldsymbol{V}_{2}$, the horizontal and vertical velocities at the free interface $z_{2}$, can be computed as

$$
V_{2}=\boldsymbol{T} \boldsymbol{U}^{-1}
$$

and the reflection coefficients matrix is

$$
\boldsymbol{R}_{m, 2}^{\mathrm{u}}=\boldsymbol{D} \boldsymbol{U}^{-1} .
$$

When a specific horizontal slowness $p$ and an angular frequency $\omega$ accidentally satisfy the Rayleigh dispersion equation and produce a surface wave mode for the truncated medium between the free surface and the $i$ th layer, the $\boldsymbol{x}_{i}(1: 2,1: 2)$ becomes singular. Such combination of $p$ and $\omega$ values must be avoided in the above approach. An example of synthetic $\mathrm{P}$ and $\mathrm{SV}$ receiver functions using the above algorithm is shown in Figure 1, and the Earth model is given in Table 1.

Table 1 A four-layer model for computing receiver functions and dispersion curves

\begin{tabular}{cccc}
\hline $\begin{array}{c}v_{\mathrm{P}} / \\
\left(\mathrm{km} \cdot \mathrm{s}^{-1}\right)\end{array}$ & $\begin{array}{c}v_{\mathrm{S}} / \\
\left(\mathrm{km} \cdot \mathrm{s}^{-1}\right)\end{array}$ & $\begin{array}{c}\text { Density/ } \\
\left(\mathrm{g} \cdot \mathrm{cm}^{-3}\right)\end{array}$ & $\begin{array}{c}\text { Thickness/ } \\
\mathrm{km}\end{array}$ \\
\hline 6.2 & 3.6 & 2.80 & 13.0 \\
6.4 & 3.6 & 2.85 & 12.0 \\
6.8 & 3.8 & 2.95 & 12.0 \\
8.2 & 4.7 & 3.40 & 0.0 \\
\hline
\end{tabular}

For linearized inversion of receiver functions, the differential receiver functions which are generated from perturbed models are required. If the velocity in a layer is changed from $v_{i}$ to $v_{i}+\delta v$ and the parameters in other layers remain unperturbed, the intermediate results from the top-down approach can be used to compute the response of the layers above the perturbed layer, and the following bottom-up approach provides the necessary intermediate information for the layers below the perturbed layer. The results of two directions can be combined together to calculate the differential receiver function efficiently as done by Randall (1989) with Kennett's method. In the program-ready procedure of the bottom-up approach, two series of matrix multiplications, that is, $\boldsymbol{H}\left(z_{2}, z_{3}\right) \cdots \boldsymbol{H}\left(z_{m-1}, z_{m}\right) \boldsymbol{W}_{m} \boldsymbol{x}_{m}$ and $\boldsymbol{H}\left(z_{2}, z_{3}\right) \cdots \boldsymbol{H}\left(z_{m-1}, z_{m}\right) \boldsymbol{W}_{m} \boldsymbol{y}_{m}$ are performed from right to left simultaneously, with $\boldsymbol{x}_{m}$ and $\boldsymbol{y}_{m}$ in equation (36).

1) Start the propagation with

$$
\begin{aligned}
& \boldsymbol{x}_{m}=\boldsymbol{W}_{m}\left(\begin{array}{ll}
0 & 0 \\
0 & 0 \\
1 & 0 \\
0 & 1
\end{array}\right), \\
& \boldsymbol{y}_{m}=\boldsymbol{W}_{m}\left(\begin{array}{ll}
1 & 0 \\
0 & 1 \\
0 & 0 \\
0 & 0
\end{array}\right),
\end{aligned}
$$



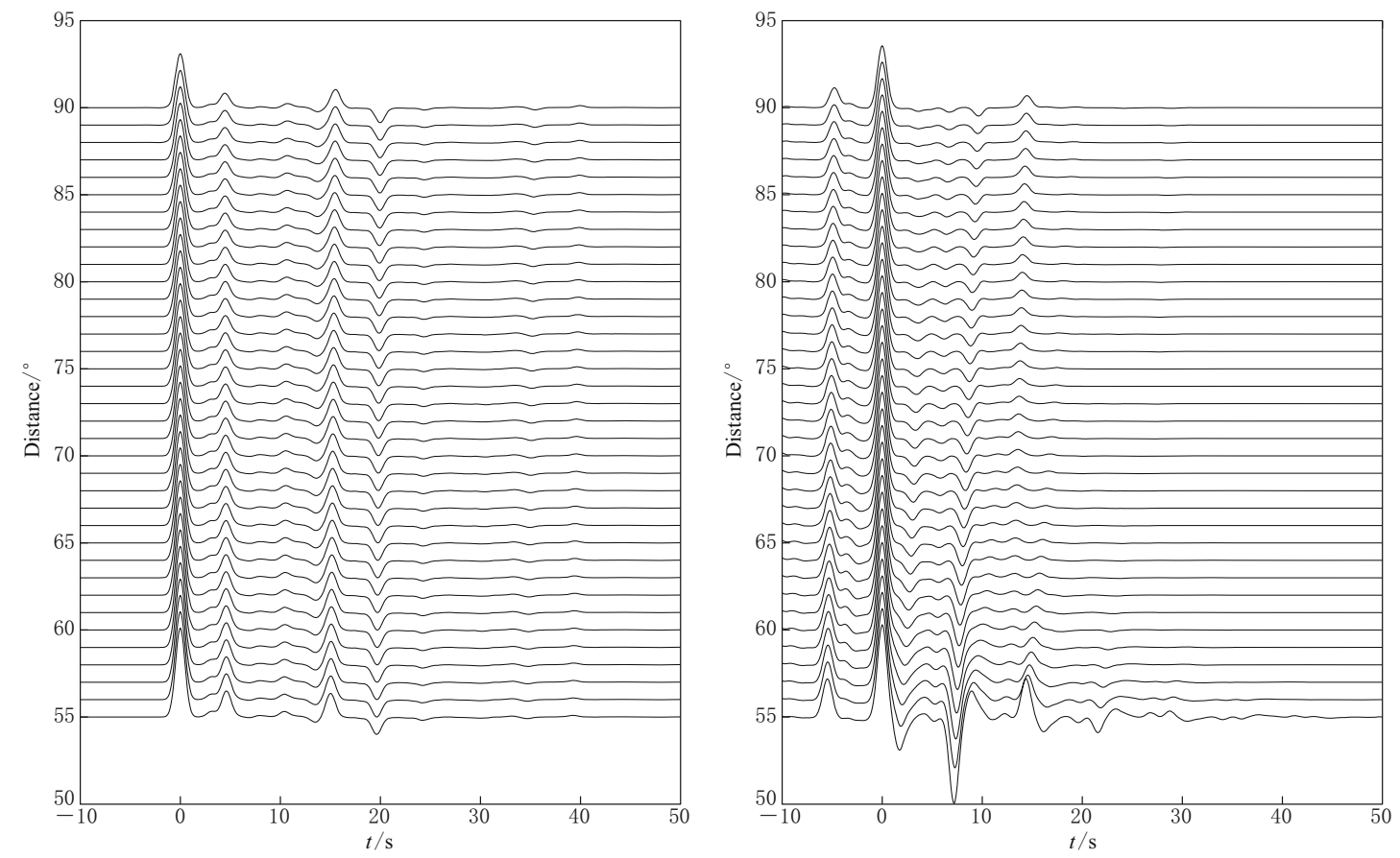

Figure 1 Synthetic receiver functions.

$$
\boldsymbol{T}=\left(\begin{array}{ll}
1 & 0 \\
0 & 1
\end{array}\right)
$$

2) Loop for $i=m-1$ down to 2

$$
\begin{aligned}
& \boldsymbol{x}_{i} \leftarrow \boldsymbol{L}_{i}^{-1} \boldsymbol{x}_{i+1} \\
& \boldsymbol{y}_{i} \leftarrow \boldsymbol{L}_{i}^{-1} \boldsymbol{y}_{i+1}
\end{aligned}
$$

(a) if $p<\frac{1}{\alpha_{i}}$ then $\quad \boldsymbol{x}_{i} \leftarrow \boldsymbol{X}_{i} \boldsymbol{x}_{i} ; \quad \boldsymbol{y}_{i} \leftarrow \boldsymbol{X}_{i} \boldsymbol{y}_{i}$

(b) else $\quad \boldsymbol{x}_{i} \leftarrow\left(\boldsymbol{W}_{i}^{-1} \boldsymbol{L}_{i}\right) \boldsymbol{x}_{i} ; \quad \boldsymbol{y}_{i} \leftarrow\left(\boldsymbol{W}_{i}^{-1} \boldsymbol{L}_{i}\right) \boldsymbol{y}_{i}$; build a $2 \times 2$ matrix $\boldsymbol{Q}=\boldsymbol{x}_{i}(3: 4,1: 2)^{-1} ; \boldsymbol{x}_{i} \leftarrow \boldsymbol{x}_{i} \boldsymbol{Q} ; \boldsymbol{y}_{i} \leftarrow$ $\boldsymbol{y}_{i}-\boldsymbol{x}_{i} \boldsymbol{y}_{i}(3: 4,1: 2) ; \boldsymbol{x}_{i}(1: 2,1: 2) \leftarrow \boldsymbol{E}_{i}^{\mathrm{u}} \boldsymbol{x}_{i}(1: 2,1: 2) \boldsymbol{E}_{i}^{\mathrm{u}} ; \boldsymbol{T} \leftarrow$ $\boldsymbol{E}_{i}^{\mathrm{u}} \boldsymbol{y}_{i}(1: 2,1: 2) \boldsymbol{T} ; \quad \boldsymbol{y}_{i}(1: 2,1: 2) \leftarrow \boldsymbol{I} ; \quad \boldsymbol{x}_{i} \leftarrow\left(\boldsymbol{L}_{i}^{-1} \boldsymbol{W}_{i}\right) \boldsymbol{x}_{i} ;$ $\boldsymbol{y}_{i} \leftarrow\left(\boldsymbol{L}_{i}^{-1} \boldsymbol{W}_{i}\right) \boldsymbol{y}_{i}$

$$
\begin{aligned}
& \boldsymbol{x}_{i} \leftarrow \boldsymbol{L}_{i} \boldsymbol{x}_{i} \\
& \boldsymbol{y}_{i} \leftarrow \boldsymbol{L}_{i} \boldsymbol{y}_{i}
\end{aligned}
$$

3) Determine the velocities at the free surface $z_{2}$ from the partitions of $\boldsymbol{x}_{2}$ and $\boldsymbol{y}_{2}$

$$
\begin{gathered}
\boldsymbol{y}_{2} \leftarrow \boldsymbol{y}_{2} \boldsymbol{T} \\
\boldsymbol{V}_{2} \leftarrow \boldsymbol{y}_{2}(1: 2,1: 2)-\boldsymbol{x}_{2}(1: 2,1: 2) \\
\boldsymbol{x}_{2}(3: 4,1: 2)^{-1} \boldsymbol{y}_{2}(3: 4,1: 2),
\end{gathered}
$$

where $\boldsymbol{I}$ is the $2 \times 2$ identity matrix. In fact, just as shown in the previous section 4.1 , the $\boldsymbol{x}_{i}(1: 2,1: 2)$, after orthogonalized by $\boldsymbol{Q}$, is the generalized reflection coefficients $\boldsymbol{R}_{i+1, m}^{\mathrm{d}}$, and the $\boldsymbol{y}_{i}(1: 2,1: 2)$ in this procedure, after the lower submatrix $\boldsymbol{y}_{i}(3: 4,1: 2)$ is eliminated to zeros, becomes $\boldsymbol{T}_{i+1, m}^{\mathrm{u}}$, that is, the generalized transmission coefficients between the interfaces $z_{i+1}$ and $z_{m}$ with incident of up-going waves at $z_{m}$.

\subsection{Rayleigh wave dispersion}

Here the layer system is the same as that in section 4.2. The product of $\boldsymbol{H}\left(z_{2}, z_{3}\right) \cdots \boldsymbol{H}\left(z_{m-1}, z_{m}\right) \boldsymbol{W}_{m} \boldsymbol{x}_{m}$ is calculated in the exactly same way as the procedure of section 4.2 with only $\boldsymbol{x}_{m}$ in equation (36). After the first two steps of the above procedure are finished, the resultant matrix $\boldsymbol{x}_{2}$ can be written as

$$
\left(\begin{array}{c}
\boldsymbol{U} \\
\boldsymbol{D}
\end{array}\right)=\boldsymbol{H}\left(z_{2}, z_{3}\right) \cdots \boldsymbol{H}\left(z_{m-1}, z_{m}\right) \boldsymbol{W}_{m}\left(\begin{array}{cc}
0 & 0 \\
0 & 0 \\
1 & 0 \\
0 & 1
\end{array}\right) \boldsymbol{Q}_{k_{1}} \boldsymbol{E}_{k_{1}}^{\mathrm{u}} \boldsymbol{Q}_{k_{2}} \boldsymbol{E}_{k_{2}}^{\mathrm{u}} \cdots \boldsymbol{Q}_{k_{n}} \boldsymbol{E}_{k_{n}}^{\mathrm{u}}
$$


The dispersion equation can be derived from equation (37) above, i.e.,

$$
\operatorname{det}\left[\boldsymbol{D}\left(\boldsymbol{Q}_{k_{1}} \boldsymbol{E}_{k_{1}}^{\mathrm{u}} \boldsymbol{Q}_{k_{2}} \boldsymbol{E}_{k_{2}}^{\mathrm{u}} \cdots \boldsymbol{Q}_{k_{n}} \boldsymbol{E}_{k_{n}}^{\mathrm{u}}\right)^{-1}\right]=0,
$$

where $\operatorname{det}[\cdot]$ indicates the operation of determinant. Since the matrices $\boldsymbol{Q}_{k_{i}}$ and $\boldsymbol{E}_{k_{i}}^{\mathrm{u}}$ are invertible, they can be ignored in equation (38). However it is helpful to keep their phases to ensure the final determinant in equation (38) remains real-valued (Buchen and Ben-Hador, 1996), i.e.,

$$
\operatorname{det}[\boldsymbol{D}] \mathrm{e}^{-\mathrm{i} \arg \left(\operatorname{det}\left[\boldsymbol{Q}_{k_{1}} \boldsymbol{E}_{k_{1}}^{\mathrm{u}} \boldsymbol{Q}_{k_{2}} \boldsymbol{E}_{k_{2}}^{\mathrm{u}} \cdots \boldsymbol{Q}_{k_{n}} \boldsymbol{E}_{k_{n}}^{\mathrm{u}}\right]\right)}=0,
$$

which can also eliminate the possible underflow of $\operatorname{det}\left[\boldsymbol{E}_{k_{i}-1}^{\mathbf{u}}\right]$. The phase and group velocities of the fundamental-mode Rayleigh waves for the four-layer model given in Table 1 are shown in Figure 2.

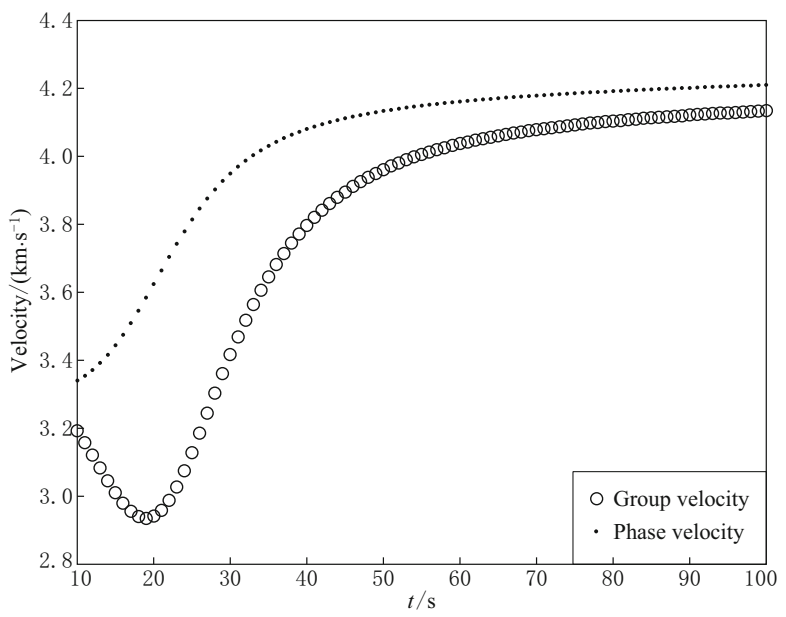

Figure 2 Dispersion of fundamental-mode Rayleigh waves.

\section{Conclusions}

The three methods, that is, the orthonormalization technique, the second-order minor method and the reflection-transmission matrix method, as shown in this note, are closely related and can resolve the numerical difficulties equally well. Especially the orthonormalization technique and Kennett's recursive algorithm are essentially equivalent in mathematics when the numerical problems are tackled.

The combination of the orthonormalization method and the Langer block-diagonal decomposition technique provides a simple, efficient and stable algorithm for calculating the plane wave response for a stratified media. The method is completely robust for high frequencies, large layer thicknesses and horizontal slownesses. It performs the orthogonalization if necessary, and preserves the multiplications with the decomposed propagators if possible. In addition to some orthogonalizing and scaling matrices which are multiplied from right, the computing procedure of the method is still under the formalism of the Thomson-Haskell propagator method. The method is extremely simple to be implemented in computer codes to calculate reflectiontransmission coefficients, body wave receiver functions and Rayleigh wave dispersion.

Acknowledgements Part of this work was conducted during R. Wang's visit to the Graduate University of the Chinese Academy of Sciences. This study is finanical supported by National Natural Science Foundation of China (Nos. 40374009 and 40574024).

\section{References}

Aki K and Richards P G (2002). Quantitative Seismology. 2nd ed. University Science Books, Sausalito, California, 261-269.

Buchen P W and Ben-Hador R (1996). Free-mode surfacewave computations. Geophys J Int 124: 869-887.

Chapman C H (2003). Yet another elastic plane-wave, layermatrix algorithm. Geophys J Int 154: 212-223.

Chapman C H and Orcutt J A (1985). The computation of body wave synthetic seismograms in laterally homogeneous media. Rev Geophys 23(2): 105-163.

Chapman C H and Phinney R A (1972). Diffracted seismic signals and their numerical solution. Meth Comp Phys 12: $165-230$.

Dunkin J W (1965). Computation of modal solutions in layered, elastic media at high frequencies. Bull Seismol Soc Am 55(2): 335-358.

Ivansson S (1993). Delta-matrix factorization for fast propagation through solid layers in a fluid-solid medium. $J$ Comp Phys 108: 357-367.

Kennett B L N (1974). Reflections, rays and reverberations. Bull Seismol Soc Am 64(6): 1 685-1 696.

Kennett B L N (1983). Seismic Wave Propagation in a Stratified Medium. Cambridge University Press, Cambridge, 25-31, 126-136.

Kennett B L N (2001). The Seismic Wavefield. Vol I: Introduction and Theoretical Development. Cambridge University Press, Cambridge, 208-215, 260-270.

Kind R and Odom R I (1983). Improvements to layer matrix methods. J Geophys 53: 127-130.

Müller G (1985). The reflectivity method: A tutorial. J Geophys 58: 153-174.

Panza G F (1985). Synthetic seismograms: the Rayleigh waves modal summation. $J$ Geophys 58: 125-145.

Pitteway M L V (1965). The numerical calculation of wavefields, reflection coefficients and polarizations for long 
radio waves in the lower ionosphere. Phil Trans $R$ Soc Lond A 257(1079): 219-241.

Randall G E (1989). Efficient calculation of differential seismograms for lithospheric receiver functions. Geophys $J$ Int 99: 469-481.

Thrower E N (1965). The computation of the dispersion of elastic waves in layered media. J Sound Vib 2(3): 210226.

Wang R (1999). A simple orthonormalization method for stable and efficient computation of Green's functions. Bull Seismol Soc Am 89: 733-741.

Wang R and Kümpel H-J (2003). Poroelasticity: efficient modeling of strongly coupled, slow deformation processes in a multilayered half-space. Geophysics 68(2): 705-717.
Wang R, Lorenzo-Martín F and Roth F (2003). Computation of deformation induced by earthquakes in a multi-layered elastic crust - FORTRAN programs EDGRN/EDCMP. Comp Geosc 29: 195-207.

Wang R, Lorenzo-Martín F and Roth F (2006). PSGRN/PSCMP: A new code for calculating co- and postseismic deformation, geoid and gravity changes based on the viscoelastic-gravitational dislocation theory. Comp Geosc 32: 527-541.

Woodhouse J H (1980). Efficient and stable methods for performing seismic calculations in stratified media. In: Dziewonski A M and Boschi E eds. Proc. Int. School of Physics 'Enrico Fermi', Course LXXVIII, Physics of the Earth's Interior. North-Holland, Amsterdam, 127-151. 